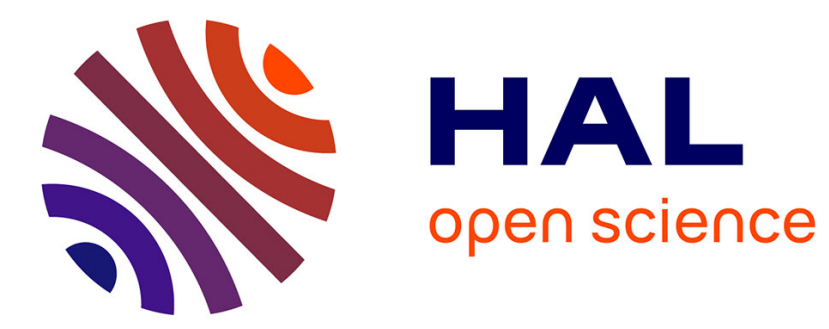

\title{
Joint Routing and Energy Management in UnderWater Acoustic Sensor Networks
}

Fatma Bouabdallah, Chaima Zidi, Raouf Boutaba

\section{To cite this version:}

Fatma Bouabdallah, Chaima Zidi, Raouf Boutaba. Joint Routing and Energy Management in UnderWater Acoustic Sensor Networks. IEEE Transactions on Network and Service Management, 2017, 14 (2), pp.456-471. 10.1109/TNSM.2017.2679482 . hal-01591952

\section{HAL Id: hal-01591952 https://hal.science/hal-01591952}

Submitted on 22 Sep 2017

HAL is a multi-disciplinary open access archive for the deposit and dissemination of scientific research documents, whether they are published or not. The documents may come from teaching and research institutions in France or abroad, or from public or private research centers.
L'archive ouverte pluridisciplinaire HAL, est destinée au dépôt et à la diffusion de documents scientifiques de niveau recherche, publiés ou non, émanant des établissements d'enseignement et de recherche français ou étrangers, des laboratoires publics ou privés. 


\title{
Joint Routing and Energy Management in UnderWater Acoustic Sensor Networks
}

\author{
Fatma Bouabdallah, Chaima Zidi, and Raouf Boutaba, Fellow, IEEE
}

\begin{abstract}
Interest in UnderWater Acoustic Sensor Networks (UW-ASNs) has rapidly increased with the desire to control the large portion of the world covered by oceans. Fundamental differences between underwater acoustic propagation and terrestrial radio propagation may impose the design of new networking protocols and management schemes. In this paper, we focus on these fundamental differences in order to conceive a balanced routing strategy that overcomes the energy holes problem. Indeed, energy management is one of the major concerns in UW-ASNs due to the limited energy budget of the underwater sensor nodes. In this paper, we tackle the problem of energy holes in UW-ASNs while taking into consideration the unique characteristics of the underwater channel. The main contribution of this study is an in-depth analysis of the impact of these unique underwater characteristics on balancing the energy consumption among all underwater sensors. We prove that we can evenly distribute the transmission load among sensor nodes provided that sensors adjust their communication power when they send or forward the periodically generated data. In particular, we propose a balanced routing strategy along with the associated deployment pattern that meticulously determines the load weight for each possible next hop, that leads to fair energy consumption among all underwater sensors. Consequently, the energy holes problem is overcome and hence the network lifetime is improved.
\end{abstract}

Index Terms-UnderWater Acoustic Sensor Networks, routing, load management, energy management, performance analysis.

\section{INTRODUCTION}

$\mathbf{T}$ HE growing interest in the design of underwater networks is motivated by the wish to provide autonomous support for many activities such as offshore exploration, tsunami warning, and mine reconnaissance. Consequently, UnderWater Acoustic Sensor Networks (UW-ASNs) are gaining a remarkable momentum within the research community. Acoustic communication is deemed to be the enabling technology for underwater networks. Indeed, radio technology is unsuitable for underwater environments due to its poor propagation through water. Optical waves require the transmitter and receiver to be aligned in order to form a link and tend to be effective at very short range compared to the desired communication distances. Consequently, acoustic modems are the current technology of choice for underwater communications.

Conceiving network protocols especially tailored for underwater acoustic networks faces serious challenges. Indeed, underwater channel imposes unique and harsh characteristics such as the high-attenuation, bandwidth-limited underwater

C. Zidi is the corresponding author, E-mail: czidi@kau.edu.sa. She is with LIPADE Laboratory, Paris Descartes University, France.

F. Bouabdallah and C. Zidi also are with Faculty of Computing and Information Technology, King Abdulaziz University, P.O. Box 42808, Jeddah.

R. Boutaba is with School of Computer Science, University of Waterloo ; 200 University Ave. W., Waterloo, ON, Canada. acoustic channel and limited battery power. In fact, batteries of underwater sensors are not only limited in terms of budget but most importantly cannot be easily recharged, since for instance solar energy cannot be exploited. Moreover, acoustic underwater communications consume a larger amount of power compared to the terrestrial radio ones. Indeed, underwater communication is subject to transmission over higher distances and more complex signal processing techniques are required at receivers to compensate the impairments of the underwater channel.

Due to the aforementioned reasons, UW-ASNs require protocols that make judicious use of the limited energy capacity of the underwater sensor nodes. To this end, one of the major characteristics of UW-ASNs that should be appropriately exploited in order to enhance the network management in terms of energy expenditure and transmission delay is their manual deployment. Underwater sensors are manually bottom anchored meaning that a prior knowledge of their locations can be acquired at deployment time. As such, we can take advantage of this feature in order to achieve a dedicated well studied deployment that satisfies our application requirements especially in terms of energy conservation.

Once the target deployment is achieved, another crucial way that can be exploited to extend the lifespan of an UWASN is load management. The goal is that all the sensors consume their energy budget as smoothly and uniformly as possible. In terrestrial wireless sensor networks, it was shown that the closest sensors to the sink tend to deplete their provided amount of energy faster than other sensors [14]- [17]. This unbalanced energy consumption is liable to drastically reduce the lifetime of sensor networks; that is why it should be avoided to the largest possible extent. In fact, authors in [17] showed that by the time the nearest sensors to the sink drain their initially provided energy, sensors more distant still have up to $93 \%$ of their energy budget. Indeed, sensors in the vicinity of a static sink act as the traffic hot spots since they have significant packet load to relay. Those sensors which are 1-hop away from a static sink would suffer from a severe exhaustion of their battery power, which may cause energy holes resulting in possible network disconnection and consequently preventing reports from reaching the sink.

In this paper, a balanced routing design for avoiding energy holes in UW-ASNs is proposed and thoroughly evaluated. Our ultimate aim is to balance the energy consumption among all underwater sensors which are manually deployed according to a defined deployment pattern such that network management is facilitated. Our balanced routing solution dictates that each underwater sensor can tune its transmission power among multiple possible levels. Each transmission power allows the 
sensor to reach possibly a specified next hop. We strive for deriving the optimal load weight for each possible power level that leads to fair energy consumption among all sensors in the network and hence the sink-hole problem is overcome. Our proposed routing scheme is especially tailored for the underwater environment. Indeed, our routing solution takes into consideration the unique characteristics of the underwater channel through the use of the time-varying channel gain derived in [6].

Our contributions can be summarized as follows. First, we propose a designed deployment pattern for UW-ASNs aimed at balancing the energy consumption and hence an improved overall energy management. Second, based on the proposed deployment, we prove that we can evenly distribute the transmission load among underwater sensors with constant data reporting provided that sensors adjust their transmission powers when they send or forward sensed data. In particular, we assume that each sensor can dynamically adjust its transmission power up to a predefined number of levels $n$. Consequently, at the routing layer, for each value of $n$, we determine the set of possible next hops with the associated load weight that lead to fair energy depletion among all sensors in the network. Third, we derive the optimal number of transmission power levels, $n$, that maximizes the network lifetime by overcoming the energy holes problem. In fact, there clearly exists a compromise between the number of transmission power levels $(n)$ and the energy consumption [19], [21]. On one hand, as we increase $n$, we allow the traffic load to be much more distributed among all the upstream coronas and hence a balanced traffic load distribution is achieved resulting in a balanced energy consumption throughout the network. On the other hand, increasing $n$ raises the energy consumption since the farthest coronas may be reached. Consequently, there clearly exists an optimal $n$ value for which the network lifetime is maximized. Finally, we prove that our balanced routing design outperforms the nominal communication range based data forwarding [24] in terms of energy conservation and hence the network lifetime. Recall that our proposed routing solution is especially designed for the underwater environment since it takes into account the fundamental characteristics of the underwater acoustic propagation. That being said, it is worth pointing out that our proposed routing protocol is more suitable for collision-free MAC protocols like [4], [5].

This paper is organized as follows. Section II presents the state of the art related to the focus of this paper. Section III presents the system model to be studied. Section IV formulates and analytically solves the energy balancing problem. Specifically, we solve a linear optimization problem that leads to an even energy depletion among all sensors. The results are provided in Section $\mathrm{V}$, where we compare the performance of our proposal to the nominal transmission range based data forwarding scheme. This paper concludes with a summary of our conclusions and contributions.

\section{RELATED WORK}

The unique characteristics of the underwater channel usually necessitate dedicated solutions. While extensive work has been conducted up to date at the physical layer [6]- [13], less work has been done at higher layers of the protocol stack and thus there is still room for innovation. Authors in [18], [2] and [1] provide an overview of existing networking protocols for underwater networks. In this section, we mainly focus on the work related to routing in UW-ASNs and energy sink-hole problem in terrestrial sensor networks. In Table 1, we provide a summary of the characteristics of the already proposed protocols related to our work and described below.

\section{A. Routing in $U W-A S N s$}

Geographical routing protocols seem appropriate for the underwater environment, where manually anchored nodes have knowledge of their coordinates at deployment time, and mobile nodes (such as AUVs) have local navigation systems. Several geographical routing protocols, especially devised for underwater channel have been proposed. In [19], the design of minimum energy routing protocols especially designed for the underwater environment is evaluated. The authors in [19] prove that, depending on the modem performance, in dense networks there is an optimal number of hops beyond which the system performance, especially in terms of energy consumption, does not improve. In [20], two distributed routing strategies are proposed for delay-insensitive and delay-sensitive applications. In [21], a new geographical routing strategy for underwater acoustic networks is introduced and joined with power control. The main contribution of this routing scheme called FBR is to dynamically establish routes on demand without damaging the network performance. In [22], the authors were mainly interested in providing a reliable routing solution especially dedicated for time-critical applications in underwater acoustic networks. To this end, they proposed a multipath routing scheme based on continuous power control aimed at minimizing the energy consumption without compromising the end-to-end delay. While providing a major improvement in terms of data reliability and error recovery, crucial issues such as energy consumption during reception of a packet were not taken into account in this analysis. In [23], a mathematical framework for cross-layer optimization is stated along with an associated protocol. Based on the unique properties of the underwater environment, the proposed solution provides a joint optimization among different layers. In particular, the proposed strategy allow each underwater node to jointly select its best next relay, the optimal transmission power and the error correction technique that minimize energy consumption. However, the lack of an acoustic transceiver able to dynamically adapt its parameters to instantaneously fit the link conditions limits the applicability of this approach in practice.

\section{B. Energy Sink-hole problem}

The energy sink-hole was originally addressed by Guo et al. in [27]. They proposed an energy-balanced transmission scheme that adjusts the ratio between direct transmission to the sink and next-hop transmission. Accordingly, sensor nodes are deployed in a circular disk around the sink. Each node can send a percentage of data directly to the sink and 


\begin{tabular}{|c|c|c|c|c|}
\hline & & Achievements & Technique & Limitation \\
\hline \multirow{4}{*}{$\begin{array}{l}\text { Energy- } \\
\text { Efficient } \\
\text { Routing } \\
\text { in UW- } \\
\text { ASNs }\end{array}$} & [20] & $\begin{array}{l}\text { Two distributed routing schemes for time sensi- } \\
\text { tive and insensitive applications. }\end{array}$ & $\begin{array}{l}\text { Selecting the best next to meet application- } \\
\text { dependent requirements. }\end{array}$ & $\begin{array}{l}\text { No mobility consider- } \\
\text { ation. }\end{array}$ \\
\hline & [21] & $\begin{array}{l}\text { A routing scheme (FBR) establishing on demand } \\
\text { routes while varying transmit power level. }\end{array}$ & $\begin{array}{l}\text { Selecting the next relay from candidates nodes } \\
\text { (reached with the used power level) }\end{array}$ & $\begin{array}{l}\text { No specific criteria to } \\
\text { select the next hop. }\end{array}$ \\
\hline & [22] & $\begin{array}{l}\text { A multipath routing scheme for time critical } \\
\text { applications. }\end{array}$ & $\begin{array}{l}\text { Selecting the optimal path from the established } \\
\text { paths. }\end{array}$ & $\begin{array}{l}\text { Routes establishment } \\
\text { overhead. }\end{array}$ \\
\hline & [23] & $\begin{array}{l}\text { A mathematical farmework for cross-layer opti- } \\
\text { mization and energy consumption minimization. }\end{array}$ & $\begin{array}{l}\text { Selecting the best next relay, the optimal trans- } \\
\text { mission power, the error correction. }\end{array}$ & $\begin{array}{l}\text { Routes establishment } \\
\text { overhead. }\end{array}$ \\
\hline \multirow{6}{*}{$\begin{array}{l}\text { Sink-hole } \\
\text { problem } \\
\text { solutions }\end{array}$} & [27] & $\begin{array}{l}\text { A hybrid data transmission protocol to mitigate } \\
\text { energy holes. }\end{array}$ & $\begin{array}{l}\text { Adjusting the ratio between transmission modes } \\
\text { (single hop and multihop). }\end{array}$ & \multirow{6}{*}{$\begin{array}{l}\text { non-suitable for UW- } \\
\text { ASNs } \\
\text { unless upgraded. }\end{array}$} \\
\hline & [25] & $\begin{array}{l}\text { An analytical model for multipath propagation } \\
\text { to distribute energy consumption among all sen- } \\
\text { sors. }\end{array}$ & $\begin{array}{l}\text { Distributing the traffic load of each sensor node } \\
\text { through multiple paths. }\end{array}$ & \\
\hline & [29] & $\begin{array}{l}\text { A virtual network partition into coronas to min- } \\
\text { imize each path'energy consumption. }\end{array}$ & $\begin{array}{l}\text { Computing of the number of hops and the opti- } \\
\text { mal width of coronas. }\end{array}$ & \\
\hline & [30] & $\begin{array}{l}\text { A non uniform nodes deployment solutions to } \\
\text { avoid sink-hole problem. }\end{array}$ & Deploying more 1 hop nodes around the sink. & \\
\hline & [26] & Variable transmission range protocol. & $\begin{array}{l}\text { Increasing the transmission range to bypass ob- } \\
\text { stacles or faulty sensors. }\end{array}$ & \\
\hline & [24] & $\begin{array}{l}\text { Variable transmission range protocol to avoid } \\
\text { sink-hole problem. }\end{array}$ & $\begin{array}{l}\text { Considering sink mobility and energy hetero- } \\
\text { geneity among sensors. }\end{array}$ & \\
\hline
\end{tabular}

TABLE I

COMPARISON OF THE STATE-OF-THE-ART WORK.

the rest to the next hop. Precisely, the authors show that sensors far away from the sink should send a larger percentage of data to the next hop, while sensors near the sink send more data directly to the sink. In [25], the authors proposed a thorough analytical model for multipath propagation that evenly distributes the energy consumption among all sensors. Indeed, they show that sending the traffic generated by each sensor node through multiple paths instead of a single best path allows performance improvement especially in terms of energy consumption. Accordingly, they derive the set of paths to be used by each sensor node and the associated proportion of utilization that minimize the energy consumption. In [28], event-driven applications in a non-uniform sensor distribution were considered. The authors proposed a blind algorithm that overcomes the energy-balancing problem without a prior knowledge of the occurrences of the events. In [29], authors proved that minimizing the total amount of energy along a path is only achieved when the coronas of a circular field have the same width. Unfortunately, such configuration would inevitably lead to uneven energy depletion among sensors. Consequently, they computed the optimal widths of coronas and their optimal number in order to achieve fair energy depletion of sensors. In [30], authors revealed that up to $90 \%$ of the initially provided energy budget is unused especially in static WSN model where the sensors are uniformly distributed. For this reason, they proposed a non-uniform sensor distribution strategy and showed by simulation that it can increase the total amount of sensed data. In [26] a protocol, called Variable Transmission Range Protocol (VTRP) was proposed with the aim to overcome the energy holes problem by varying the transmission power. Indeed, VTRP proposes to dynamically adapt the transmission range such that the closest sensors to the sink are bypassed and hence the network lifetime is increased. While VTRP assumes that the sink is static, in [24] the proposed protocol considers sink mobility and energy hetero- geneity among sensor nodes in order to overcome the sink-hole problem. Different from the contributions described in this section, in this paper, we present a routing solution dedicated for a specific underwater acoustic network deployment, which overcomes the energy holes problem by achieving a fair load distribution, balanced energy consumption, and better overall network management. Note that, our routing solution takes into account the unique fundamental features of the underwater environment. Indeed, most of the early work on routing layer has focused on dealing with long delays while providing energy efficient communication but has not included important propagation factors such as frequency-dependant attenuation, bottom surface reflections and Doppler effect, which highly affect energy consumption through both power and rate. A critical component for the development of routing protocols is the understanding of the impacts of channel properties on key metrics used for routing such as energy consumption and delay. Preliminary work on capacity was presented in [32], which defines the impact of the bandwidth-distance relationship on link bit rate power but does not directly extend to a multihop scenario. As a distinguishing feature from the abovedescribed works, in our study, each sensor node has multiple possible transmission ranges. As such, each sensor node has multiple possible next hops to reach the sink. Consequently, we strive for deriving the appropriate load weight for each possible next hop such that the energy depletion is balanced among all sensors in the network. We believe that the proposed balanced routing design is able to efficiently overcome the energy holes problem while still remaining practical enough for real implementation.

In our study, we opt for the pre-configured routing instead of using the adaptive dynamic routing for three main reasons. First, in our study, the sensor nodes are not actually mobile since they are manually bottom anchored. Hence, the overall network topology is static. Even more, in our work, we 
propose a well-defined deployment pattern for the underwater sensors such that our balanced pre-configured routing solution perfectly achieves the application requirements especially in terms of energy savings.

The second reason behind using the pre-configured routing is the traffic pattern. In our study, we consider continuous monitoring applications, where each node reports periodically its data to the sink node. The amount of information generated by each sensor node is therefore known a priori. In fact, in typical networks, the whole network topology is not expected to change dramatically. Finally, performing dynamic routing is not recommended for the following third reason. It is well known that dynamic routing induces considerable exchange of signaling messages. This routing scheme requires global and real-time information about the network state to make routing decisions. In UW-ASNs, this can be extremely costly, since a large amount of energy has to be spent to route control packets.

\section{MODEL AND PROBLEM DEFINITION}

\section{A. Time-varying underwater channel}

1) The channel gain: In [6], an exhaustive mathematical analysis was conducted to describe the time-varying channel gain in underwater environment by taking into account most of the physical features of acoustic propagation such as frequency-dependent attenuation, the bottom surface reflections as well as the effects of inevitable random local displacements and its induced Doppler effect. The proposed analytical model was validated using experimental data and hence an underwater acoustic simulator was developed [7] to provide the instantaneous channel gain between a transmitter and a receiver, given their nominal coordinates and their displacement ranges. According to [6], underwater channel variations can be classified according to the size of the random displacement undergone by underwater sensors. Indeed, due to many phenomena (water current, transmitter/receiver drifting, surface waves...) underwater sensors may undergo displacement on the order of a few or many wavelengths. The former is referred to as small-scale variations and the latter as large-scale variations. Small-scale variations occur over short displacements and correspondingly short period of time during which the system geometry (especially in terms of nodes' locations) and environmental conditions do not go through remarkable change and hence are rather considered static. However, large-scale modeling takes into account variations caused by location shifting as well as varying environmental conditions. In other words, large-scale variations are modeled as a consequence of random system displacements spanning a given variation range leading thus to large-scale variation in the gains and delays of propagation paths. More precisely, in [6], transmitter/receiver within a nominal channel geometry have multiple propagation paths with different lengths and different angles of arrival. Large-scale variations cause each propagation path length to deviate randomly and considerably from the nominal. In addition to that, small-scale variations cause every propagation path to be further scattered into a number of micro-paths. Hence, small-scale variations influence the instantaneous channel response, and, consequently, the instantaneous signal-to-noise ratio (SNR). Therefore, small-scale

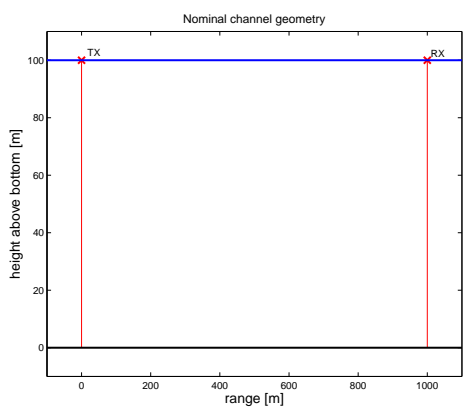

Fig. 1. Nominal Channel Geometry: distance between the transmitter and receiver is $d=1 \mathrm{~km}$, water depth $h=100 \mathrm{~m}$, transmitter and receiver are at the water surface and the bandwidth $B=10 \mathrm{Khz}$.

channel variations are to be considered during a few consecutive packet transmissions. In contrast, large-scale variations impact the average SNR over longer periods of time. That's why, they are mostly significant for the study of top-level system functions such as power allocation. Accordingly, the authors define the instantaneous channel gain for a particular realization of the large-scale displacement and a particular realization of the small-scale variation for a system operating in the frequency range $\left[f_{\min }, f_{\min }+B\right]$ as

$$
\tilde{G}(t)=\frac{1}{B} \int_{f_{\min }}^{f_{\min }+B}|H(f, t)|^{2} d f
$$

and the large-scale gain $G$ which is the locally averaged instantaneous gain over small-scale realizations as

$$
G=E\{\tilde{G}(t)\}
$$

where $H(f, t)$ represents the time-varying channel transfer function that was derived in [6]. Note that, in our work, we use the averaged instantaneous gain $G$ to compute the needed transmission power to communicate a data packet between a pair of nodes in the network. While we rather use the instantaneous channel gain $\tilde{G}(t)$ to determine if a data packet was successfully received by the intended receiver. More details are provided in the next section. For illustration purposes, we focus on an acoustic channel example whose parameters are depicted in Fig. 1. Let us assume that the transmitter and receiver are slightly displaced around their nominal locations by some heights $\Delta h_{T}, \Delta h_{R}$ and a distance $\Delta d$, and let us also assume that the surface height is displaced by some $\Delta h$. Specifically, let us suppose that all displacements are uniformly distributed, each on the interval $\pm 5 \mathrm{~m}$. Treating these displacements as random variables, we generate the instantaneous channel gain as shown in Fig. 2.

In Fig. 3, we plotted the average channel gain as a function of distance using the acoustic simulator proposed by Milica Stojanovic and Qarabaqi in [7]. The distance changes from $100 \mathrm{~m}$ to $3 \mathrm{~km}$ in steps of $200 \mathrm{~m}$. For each value of the distance, there are 150 realizations of the acoustic channel. For every realization, the transmitter and the receiver are initially placed at the water surface within a depth of $h=100 \mathrm{~m}$ and using a bandwidth of $10 \mathrm{kHz}$ to communicate. Once placed, 


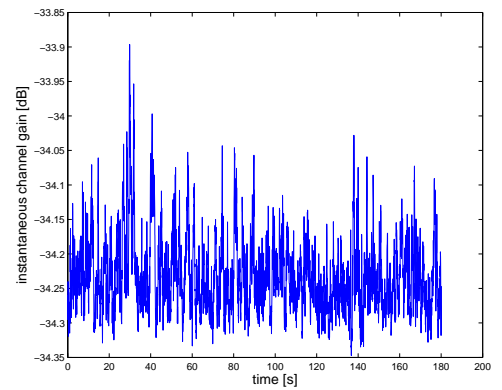

Fig. 2. Instantaneous Channel Gain.

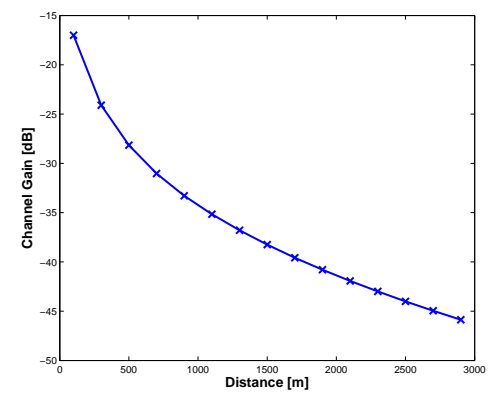

Fig. 3. The channel gain versus distance.

the transmitter, the receiver, and the water surface randomly drift from their nominal locations by some heights $\Delta h_{T}, \Delta h_{R}$ and $\Delta h$, respectively. Besides, the distance separating the transmitter from the receiver randomly change by some $\Delta d$. Specifically, we suppose that all these displacements $\left(\Delta h_{T}\right.$, $\Delta h_{R}, \Delta h$ and $\Delta d$ ) are uniformly distributed, each on the interval $\pm 5 \mathrm{~m}$. As expected, as the distance separating the transmitter from the receiver increases, the channel quality will degrade resulting in a decreasing channel gain.

2) Power Allocation: In a time-varying channel, the power allocation can be either adaptive or invariant. In the latter case, where no power control mechanism is applied a fixed large margin is to be introduced to ensure that the SNR remains above a given threshold $S N R_{0}$, regardless of the channel conditions. However, for energy efficiency reasons, the transmit power consumption can be minimized if the transmitter has at its disposal some knowledge of the channel gain as stated in [9]. Ideally, if the exact fading gain $G$ is known, the transmit power $\hat{P}_{T}$ can be rigorously tuned accordingly such that the SNR is kept at the value $S N R_{0}$. In this ideal case, and as introduced in [9], $\hat{P}_{T}$ can be simply adjusted to

$$
\hat{P}_{T}=P_{T 0} \bar{G} / G
$$

where $\bar{G}$ is the mean of the actual exact channel gain $G$ and $P_{T 0}$ simply denotes the needed power to achieve a target $S N R_{0}$ in the absence of fading $(G=\bar{G}) . P_{T 0}$ can be derived as follows:

$$
P_{T 0}=P_{N} \times S N R_{0} / \bar{G}
$$

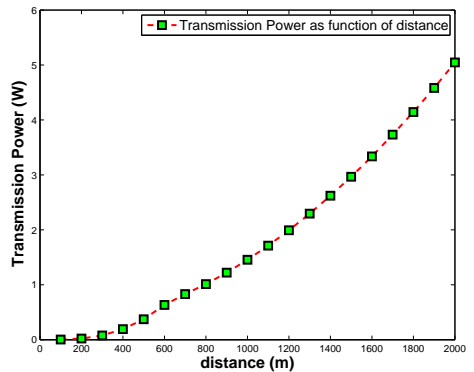

Fig. 4. Transmission power as function of distance.

where $P_{N}$ is the noise power over $B$. Note that, in such ideal case, adopting such value for $\hat{P}_{T}$ will always guarantee the successful reception of a data packet. However, as explained in [9], deriving $\hat{P}_{T}$ using $G$ can be challenging as the channel can not be fully known beforehand. Rather, an estimate $\hat{G}$ is to be used in place of the true actual gain $G$. In this case, a margin $K$ has to be introduced to guarantee to some extent that the gap between the estimated and the true channel gain does not lead to an outage. The transmit power is then adjusted according to

$$
\hat{P}_{T}=K P_{T 0} \bar{G} / \hat{G} .
$$

In our work, we suggest to consider $\hat{G}$ such that the probability that, the actual exact fading gain $G$ is less than $\hat{G}$, falls behind a given reliability level, $R_{\text {per }}$. $R_{\text {per }}$ is to be decided by the network manager depending on the application requirements. In other words, we set $\hat{G}$ such that

$$
P(G<\hat{G}) \leq R_{p e r}
$$

It is worth noting that, in order to conceive a power allocation scheme, we rather consider $G$ than $\hat{G}(t)$. Indeed, as previously explained, since large-scale variations influence the average SNR over longer periods of time, it is rather more meaningful to consider the large-scale gain $G$ to conceive a power allocation scheme. However, the instantaneous channel gain $\hat{G}(t)$ is more convenient for deriving the instantaneous signal-to-noise ratio (SNR) and hence to determine if a packet was successfully received or has encountered a failure.

Fig. 4 shows the generated transmission power as a function of distance for the acoustic channel parameters listed in the previous section. Note that, we consider a noise power of $20 \mathrm{~dB}, R_{\text {per }}$ is set equal to $85 \%$ and the margin $K$ equals 1.1. Is is worth pointing out that the transmission power is a non-linearly increasing function of distance. Note that by adjusting the transmission power according to $\hat{P}_{T}$ the channel time-variability is now taken into consideration.

Recall that, once a communication is initiated using a given transmit power $\hat{P}_{T}$, the received signal power can then be determined according to the undergone instantaneous channel gain $\tilde{G}(t)$. Let us consider a practical UWA communication between two nodes $i$ and $j$ separated by a nominal distance $d$ and operating in a frequency bandwidth $\left[f_{\min }, f_{\max }\right]$ with a width $B$. Accordingly, the transmission from $i$ to $j$ will un- 
dergo an instantaneous channel gain of $\tilde{G}(t)_{(i, j)}$. The received signal power can then be expressed as

$$
\tilde{P}_{R}(t)_{(i, j)}=\hat{P}_{T(i, j)} \tilde{G}(t)_{(i, j)}
$$

Note that, the average received signal power during the reception period of time will help us decide if a data packet was successfully received, as explained in the next section.

3) Probability of successful packet reception: In our work, in order to further take into account the time-variability of the underwater acoustic channel, we introduce a success probability $P_{j}^{i}$ over a link $(i, j)$ which represents the probability of a successful reception by a node $j$ for a transmission initiated by $i$ using a transmission power $\hat{P}_{T(i, j)}$ over a bandwidth $B$. In fact, a packet reception is considered successful by sensor $j$ for a communication initiated by $i$ during the time period $\left[t_{1}, t_{2}\right]$ if and only if

$$
\frac{1}{t_{2-} t_{1}} \int_{t_{1}+D_{i j}}^{t_{2}+D_{i j}} \frac{\tilde{P}_{R}(t)_{(i, j)}}{P_{N}} d t \geq S N R_{0}
$$

where $D_{i j}$ refers to the propagation time between $i$ and $j$. It is worth noting that $P_{j}^{i}$ is determined numerically for each link in the network using the underwater acoustic simulator developed in [7] and represents the fraction of successfully received packets. As expected, $P_{j}^{i}$ will impact the total amount of traffic received by every node in the network and thus the total energy consumption will be affected. Given that our objective is to balance the energy consumption throughout the network, $P_{j}^{i}$ along with $\hat{P}_{T(i, j)}$ will inevitably impact the load weights for every node in the network as detailed in section $\mathrm{V}$.

\section{B. Energy Sink-hole problem}

In this paper, we investigate the energy sink-hole problem in underwater acoustic sensor networks, where underwater sensors located close to the underwater sink are heavily used in forwarding sensed data to it. Extensive research efforts have been devoted to analyzing the energy sink-hole problem especially in Wireless Sensor Networks (WSNs). They all agree that the energy hole problem is unavoidable in static uniformly distributed always-on WSNs where the sensors periodically report their sensed data to a static sink using their nominal communication range [27]- [31]. For this reason, most of the already undertaken research on balancing the energy consumption is mainly on using adjustable transmission power. Indeed, by allowing each sensor to dynamically adjust its transmission power, the aim is to achieve a fair load distribution among sensors and thus the closest sensors to the sink are relieved from the relying task.

Our study of the energy sink-hole problem in UW-ASNs is motivated by the manual static deployment of underwater sensors in real-world applications, and hence, efficient solutions should be provided to tackle this problem. Our goal is to balance the energy depletion of all sensors in terms of traffic forwarding (number of transmitted packets) in order to extend the network lifetime. To this end, our approach for tackling the energy sink-hole problem is twofold: i) analyzing to what extent can perfect uniform energy depletion among all sensors in the network be assured such that the energy sink-hole problem in UW-ASNs is overcome and ii) studying how can the energy sink-hole problem in manually deployed UW-ASNs be addressed. By thoroughly investigating these two issues, we aim at closely approaching the perfect uniform energy depletion among all underwater sensors in the network.

To address the first issue, we conceive a data forwarding strategy for transmitting the periodically generated data from underwater source sensors to the sink. The goal of this forwarding scheme is to appropriately distribute the total data dissemination load on the individual underwater sensors such that the energy depletion is balanced among all sensors in the network.

To address the second issue, the set of the 1 hop away neighbors of the sink should change over time, thus allowing different subsets of sensors to act as forwarders to the sink. In other words by varying the transmission power of manually deployed sensors, the number of hops to reach the sink is continuously varying. For instance, suppose that a sensor $U$ is $2 r$ away from the sink $\mathcal{S}$. If the underwater sensor $U$ uses a transmission power to reach $r$ then $U$ is 2 hops away from $S$. However, if $U$ adopts a transmission power to reach $2 r$ then $U$ is 1-hop away from $\mathcal{S}$. Consequently, we suggest that $U$ sends a fraction of its total load using a transmission power to reach $r$ and the remaining portion will be directly sent to the sink using the appropriate transmission power for $2 r$.

To summarize, in our work, each sensor is responsible for deriving the appropriate load weight with the associated transmission power, namely potential next hop, that evenly distribute the energy consumption among underwater sensors. Moreover, we tackle the energy sink-hole problem by considering a static underwater sensor deployment strategy where underwater sensors are manually placed in a circular sensor field centered at one static sink.

\section{Network and Energy model}

The proposed deployment strategy considers a 2dimensional shallow underwater sensor network. A set of sensors is anchored to the ocean bottom and endowed with a quite long rope along with a floating buoy to push the sensor towards the surface. Indeed, the buoy can be inflated by a pump in order to push the sensor towards the ocean surface. Note that in this work, we assume that the sensors will be all the time attached to their anchors through the cable which will severely restrict their displacement. Consequently, in such scenario, these surface sensors that are bottom anchored have a complete knowledge of their geographical position at deployment time. It is worth pointing out that our proposed deployment architecture targets especially shallow water which makes the deployment cost reasonable.

In order to approach the uniform energy depletion, sensors are placed in a circular sensor field of radius $R$ centered at the sink. The sensor field is virtually partitioned into disjoint concentric sets termed coronas of constant width $r$. The width of each corona is at most $d_{t x-\max }$, the maximum transmission 


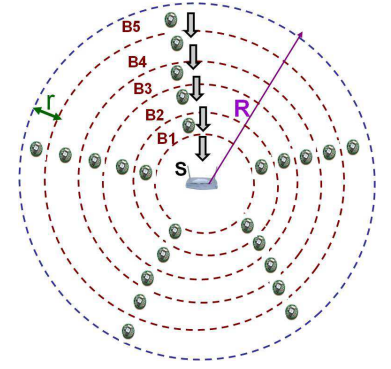

Fig. 5. Underwater Acoustic sensor network model.

range of an underwater acoustic sensor. Consider $K$ to be the number of coronas around the sink.

$$
K=\left\lfloor\frac{R}{r}\right\rfloor
$$

For example, in Fig. 5, $K=5$, hence the sensor field is partitioned into five coronas $B_{1}, B_{2}, B_{3}, B_{4}$ and $B_{5}$.

In the remainder of this paper, we consider a continuous reporting sensor application where the average number of reports generated per unit of time by each sensor node is denoted by $A$. Moreover, we assume that the energy consumption of sensors is due to data reception and transmission. In fact, since in underwater environment, the deployment is generally quite sparse, the energy depletion due to overhearing can be neglected. More precisely, the energy spent in transmitting one packet of length $P_{l}$ bits over a distance $d$ between two nodes $i$ and $j$ is given by

$$
E_{t x}(d)=\hat{P}_{T(i, j)} \times \hat{T}_{t x(i, j)}
$$

where $\hat{T}_{t x(i, j)}$ is the transmission time given by

$$
\hat{T}_{t x(i, j)}=\frac{P_{l}}{\hat{C}(d)_{(i, j)}}
$$

where $\hat{C}(d)_{(i, j)}$ is the maximum allowed capacity over the bandwidth $B$. Note that, we keep the dependance on $(d)$ for the capacity in order to emphasize that the link capacity depends on distance through the SNR.

$$
\hat{C}(d)_{(i, j)}=B \times \log _{2}\left(1+\frac{\hat{P}_{T(i, j)} \times \hat{G}_{(i, j)}}{P_{N(i, j)}}\right)
$$

Likewise, the energy spent in receiving one $P_{l}$ bits packet is given by

$$
E_{r x(i, j)}=P_{r x}{ }^{0} \times \hat{T}_{t x(i, j)}
$$

where $P_{r x}^{0}$ is the electronics power. According to the deployment pattern discussed above, routing is relatively straightforward. Each packet is forwarded from the source to the sink by crossing adjacent coronas through the immediately adjacent sensors. Figure 5 illustrates a possible path along which a packet from one sensor in the outermost corona is routed to the sink. Notice that, in this example, each hop involves the immediately adjacent neighbor from the adjacent corona. More precisely, our sensor field can be seen as a set of wedges. Each wedge $W$ is virtually partitioned into $K$ sectors, $S_{1}, S_{2}, \ldots, S_{K}$ by its intersection with $K$ concentric circles,

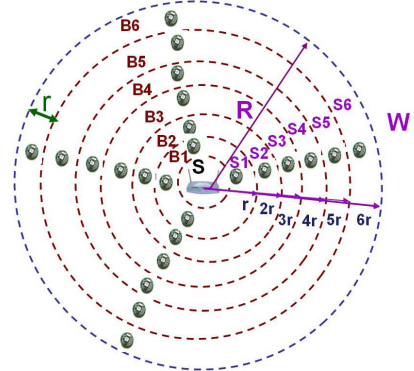

Fig. 6. A wedge $\mathrm{W}$ and the associated sectors.

centered at the sink, and of monotonically increasing radius $r, 2 r, 3 r, \ldots, K r$, as shown in Fig. 6. Each sector contains exactly one sensor which has to forward the cumulative traffic coming from its predecessors to one of its possible successors. Specifically, in our study, we assume that each sensor is capable of adjusting its transmission power in order to send the appropriate fractions of packet load to one of its possible successors within its maximum transmission range $d_{t x-\max }$. More details are given in the next section.

\section{Energy Management}

In our study, all the sensor nodes transmit periodically their reports to the sink node, denoted by $\mathcal{S}$. The average number of reports generated per unit of time by each sensor node is denoted by $A$. In this section we turn to the task of evaluating the energy expenditure per sensor in an arbitrary corona $B_{i}$ with $i \geq 1$. Observe that, according to our routing strategy, every node in a given wedge $W$ and a generic corona $B_{i}$, $(1 \leq i \leq K)$, is called upon to serve two kinds of paths:

- paths originating at an underwater sensor located in the same wedge $W$ but in a different corona $B_{j}$ with $i<j \leq K$, and

- paths emanating from the same sensor in $B_{i}$.

It is easy to show that the total number of paths that may involve a specific node in a given wedge $W$ and in corona $B_{i}$ includes all possible paths in $W$ except those originating in one of the coronas $B_{1}, B_{2}, \ldots$ and $B_{i-1}$.

For each sensor node located at corona $B_{i}$ in a specific wedge $W$, the next hop to send generated reports to the sink $\mathcal{S}$ can be the sensor located in $B_{i-1}$ or $B_{i-2}, \ldots$ or $B_{i-n}$, in the same wedge $W$ where

$$
n=\left\lfloor\frac{d_{t x-\max }}{r}\right\rfloor
$$

Considering a wedge $W$, we associate to each possible next hop located in $B_{i-1}$ or $B_{i-2}, \ldots$, or $B_{i-n}$ a respective weight $\beta_{1}^{i}, \beta_{2}^{i}, \ldots, \beta_{n}^{i}$ and a respective probability of a successful reception $P_{1}^{i}, P_{2}^{i}, \ldots, P_{n}^{i}$ such that $\sum_{p=1}^{n} \beta_{p}^{i}=1, \forall i, 1 \leq i \leq K$. Consequently, the total number of packets per unit of time, $A_{i}$, handled by a sensor in corona $B_{i}$ and wedge $W$ can simply be expressed as follows

$$
\begin{gathered}
A_{i}=A+P_{1}^{i+1} \beta_{1}^{i+1} A_{i+1} \\
+\ldots+P_{j}^{i+j} \beta_{j}^{i+j} A_{i+j}+\ldots+P_{n}^{i+n} \beta_{n}^{i+n} A_{i+n} \\
\text { if } i+j>K \text { then } \beta_{j}^{i+j}=0
\end{gathered}
$$


It is worth pointing out that, the success probability is introduced to take into account the time-variability of the underwater channel. Recall that, every pair of nodes have their own success probability which reflects the probability of successful reception on their link, as introduced in section III.A.3. By doing so along with the adjusted transmission power $\hat{P}_{T}$, the time-variability of the acoustic channel is now considered in our analytical model aiming at offline deriving the optimal load weights balancing the energy consumption throughout the network which makes the derived load weights more realistic.

Once the average load $A_{i}$ is expressed, the average transmission energy, $E_{T X}^{i}$, consumed by a sensor in corona $B_{i}$ and wedge $W$ can be derived as follows:

$$
\begin{gathered}
E_{T X}^{i}=\beta_{1}^{i} A_{i} E_{t x}(r)+\ldots+\beta_{j}^{i} A_{i} E_{t x}(j r)+\ldots+ \\
\quad \beta_{n}^{i} A_{i} E_{t x}(n r) \\
\text { if } i-j<0 \text { then } \beta_{j}^{i}=0
\end{gathered}
$$

Likewise, the average reception energy, $E_{R X}^{i}$, consumed by a sensor in corona $B_{i}$ and wedge $W$ can be expressed as follows

$$
\begin{gathered}
E_{R X}^{i}=P_{1}^{i+1} \beta_{1}^{i+1} A_{i+1} E_{r x}(r)+\ldots+P_{j}^{i+j} \beta_{j}^{i+j} A_{i+j} E_{r x}(j r) \\
+\ldots+P_{n}^{i+n} \beta_{n}^{i+n} A_{i+n} E_{r x}(n r) \\
\quad \text { if } i+j>K \text { then } \beta_{j}^{i+j}=0
\end{gathered}
$$

Finally, the total energy consumed by a sensor in corona $B_{i}$ and wedge $W$ is

$$
E^{i}=E_{T X}^{i}+E_{R X}^{i}
$$

Recall that the goal of our work is to tailor the coronas in such a way that the energy expenditure is balanced across all the coronas. Consequently, our problem can be stated as follows:

$$
\begin{aligned}
& \text { Given } K, r, d_{t x-\max } \\
& \text { Find } \beta_{1}^{i}, \beta_{j}^{i}, \ldots, \beta_{n}^{i} \forall i, 1 \leq i \leq K \\
& \text { such that } E^{1}=E^{2}=\ldots=E^{K} \\
& \text { subject to } \\
& \sum_{j=1}^{n} \beta_{j}^{i}=1, \forall i, 1 \leq i \leq K
\end{aligned}
$$

It is worth noting that the perfect uniform energy depletion is impossible to achieve. Indeed, for the derived optimization problem of Eq. (19), the number of unknowns is much greater than the number of equations. In fact, at each corona $i$ we have to determine $n$ unknown variables $\left(\left(\beta_{l}^{i}\right)_{1<l<n}\right)$ resulting on a total number of unknowns equal to $n \bar{K}$ but with only $2 K-2$ equations $\left(E^{1}=E^{2}=\ldots=E^{K}\right.$ and $\left.\sum_{j=1}^{n} \beta_{j}^{i}=1, \forall i, 1 \leq i \leq K\right)$. Consequently, our optimization problem is impossible to solve and so is the energy balancing among coronas. For this reason, we strive for approaching the optimal uniform energy depletion by determining, for each sensor node, the next possible hosts with the associated load weight that better approaches the balanced energy expenditure among underwater sensors.

In what follows, we denote $E_{r x}(j r)$ as $E_{r x}^{j}, E_{t x}(j r)$ as $E_{t x}^{j}$ and $\beta^{i}=\left(\beta_{l}^{i}\right)_{1 \leq l \leq n}$.

\section{A. Iterative Process}

We attempt to analytically approach the optimal uniform energy depletion using an iterative process. As it turns out, the $\beta^{i}$ s can be determined iteratively in a natural way. In the first iteration, we suppose that we only have the corona $B_{1}$ of width $r$. In this case, the total traffic of each sensor in $B_{1}$ is exclusively composed of the locally generated traffic $A$ and clearly $\beta_{1}^{1}$ is equal to 1 . In the second iteration, we add corona $B_{2}$ and knowing $\beta_{1}^{1}$ we try to balance the energy expenditure between $B_{1}$ and $B_{2}$ by determining $\beta_{1}^{2}$ and $\beta_{2}^{2}$. More precisely, by adding $B_{2}$, the total traffic of $B_{1}$ increases since there is a newly received traffic from $B_{2}$. Consequently, our previously established balance is perturbed. To re-arrange such imbalance we compute $\beta_{1}^{2}$ and $\beta_{2}^{2}$. Note that $\beta_{2}^{2}$ denotes the traffic weight that has to be sent directly from sensor in $B_{2}$ to the sink.

Generally speaking, suppose that we achieved iteration $j$ and hence the energy consumption between $j$ coronas is balanced. Adding corona $B_{j+1}$ will disturb the previously established balance since the total traffic in each corona will inevitably increase. Knowing $\beta^{1}, \beta^{2}, \ldots, \beta^{j}$, we settle once again our balance by determining $\beta^{j+1}$. Note that for the newly added corona $B_{j+1}, A_{j+1}=A$ and $E_{R X}^{j+1}=0$.

As we shall see shortly, $\beta^{2}$ is obtained after adding corona $B_{2}$ and as a result of writing $E^{2}=E^{1}$. By the same way, after adding corona $B_{3}, \beta^{3}$ is obtained from $E^{3}=E^{2}$ and $E^{3}=E^{1}$. More generally, at iteration $j+1, \beta^{j+1}$ is obtained from $E^{j+1}=E^{j}=\ldots=E^{1}$. The iterative process is straightforward; the details are presented next.

1) Calculation of the cumulative traffic: The cumulative traffic emanating from downstream coronas is a key variable that directly influences the energy consumption on every corona and hence the $\beta^{i}$ balancing vectors. For this reason, let us start by iteratively expressing the cumulative traffic at every corona. For this purpose, at each iteration $j+1$ (as explained above), corona $B_{j+1}$ is newly added where $A_{j+1}=A$ and thus $A_{j-k} ; \forall 0 \leq k \leq j-1$ should be newly derived since the amount of traffic $A_{j+1}$ will be forwarded to upstream coronas in order to reach the sink.

\section{Theorem 1:}

$\forall 1 \leq j+1 \leq K, A_{j+1}=A$ and $\forall 0 \leq k \leq j-1$; $A_{j-k}=\left[\alpha_{0 k}+\sum_{l=1}^{k+1} \alpha_{l k} P_{l}^{j+1} \beta_{l}^{j+1}\right] \times A$

$$
\text { where }\left\{\begin{array}{l}
\beta_{l}^{j+1}=0, \text { if } l>\min (n, k+1) \\
\alpha_{00}=1 ; \alpha_{0(-1)}=1 \\
\alpha_{0 k}=1+\sum_{m=1}^{k} \alpha_{0(k-m)} P_{m}^{j-(k-m)} \beta_{m}^{j-(k-m)} \\
\alpha_{l(-1)}=0 ; \\
\alpha_{l k}=1, \text { if } l=k+1 \\
\alpha_{l k}=\sum_{m=1}^{k+1-l} \alpha_{l(k-m)} P_{m}^{j-(k-m)} \beta_{m}^{j-(k-m)}
\end{array}\right.
$$

Theorem 1 can be proven by mathematical induction. The proof is provided in Appendix A. Note that, as expected, in Theorem $1, A_{j-k} \forall 0 \leq k \leq j-1$ linearly depends on $\beta^{j+1}$. Consequently, we aim at linearly expressing $E^{i}, \forall i$, $1 \leq i \leq j+1$, as function of $\beta^{j+1}$ and hence by writing 
$E^{j+1}=E^{i}$, a system of linear equations can be established such that the balancing vector $\beta^{j+1}$ can be determined.

2) Calculation of energy consumption in transmission and reception: Recall that our objective is to determine $\beta^{j+1}$ for each iteration $j+1$ that balance the energy consumption between $B_{1}, B_{2}, \ldots, B_{j+1}$. Consequently, at each iteration $j+1$, we strive for deriving the unknown vector $\beta^{j+1}$ of size $j+1$. Recall that, as explained above, at iteration $j+1$, all the $\beta^{i}, 1 \leq i \leq j$ are already computed. As we newly add corona $B_{j+1}$, our previously established balance will be violated and hence $\beta^{j+1}$ have to be computed such that our energy consumption balance is reinstated again. For this purpose, we aim at linearly expressing $E^{j+1}$ and $E^{i}(\forall$ $1 \leq i \leq j$ ) as function of $\beta^{j+1}$. Consequently, by writing $E^{j+1}=E^{j}=\ldots=E^{1}$ we get a system of $j+1$ equations with $j+1$ unknowns. Recall that, for the newly added corona $B_{j+1}$, $E_{R X}^{j+1}=0$ hence $E^{j+1}=E_{T X}^{j+1}$. Therefore our system will be reduced to $E_{T X}^{j+1}=E^{j}=\ldots=E^{1}$ where $E^{i}=E_{T X}^{i}+E_{R X}^{i}$ $(\forall 1 \leq i \leq j)$. $K$,

Let's start by expressing $E_{T X}^{j+1}$. By definition, $\forall 1 \leq j+1 \leq$

$$
E_{T X}^{j+1}=A \sum_{l=1}^{j+1} \beta_{l}^{j+1} E_{t x}^{l}
$$

where $\beta_{l}^{j+1}=0$ if $l>\min (n, j+1)$.

Recall that our ultimate goal is to express our problem as a system of linear equations, at each iteration.

Consequently, $E_{T X}^{j+1}$ can be linearly expressed as as function of $\beta^{j+1}$ as follows,

$$
\begin{gathered}
E_{T X}^{j+1}=\sum_{l=1}^{j+1} T X_{l}^{j+1} \beta_{l}^{j+1} \\
\text { where } T X_{l}^{j+1}=A E_{t x}^{l} .
\end{gathered}
$$

Now, let us derive $E_{T X}^{i}$ and $E_{R X}^{i}(\forall 1 \leq i \leq j)$ as function of $\beta^{j+1}$.

$E_{T X}^{i}$ can be expressed as follows

$$
E_{T X}^{i}=T X_{0}^{i}+\sum_{l=1}^{j-i+1} T X_{l}^{j-i} P_{l}^{j+1} \beta_{l}^{j+1}
$$

where $\left\{\begin{array}{l}\beta_{l}^{j+1}=0, \text { if } l>\min (n, j-i+1) \\ T X_{0}^{i}=A\left(\sum_{q=1}^{i+1} \beta_{q}^{i} E_{t x}^{q}\right) \alpha_{0(j-i)} \\ T X_{l}^{j-i}=A\left(\sum_{q=1}^{i+1} \beta_{q}^{i} E_{t x}^{q}\right) \alpha_{l(j-i)}\end{array}\right.$

Similarly, $\forall 1 \leq i \leq j, E_{R X}^{i}$ can be derived as follows

$E_{R X}^{i}=R X_{0}^{i}+\sum_{l=1}^{j-i} R X_{l}^{j-i} P_{l}^{j+1} \beta_{l}^{j+1}+P_{j-i+1}^{j+1} \beta_{j-i+1}^{j+1} A E_{r x}^{j+1-i}$

where $\left\{\begin{array}{l}\beta_{p}^{i+p}=0, \text { if } p>\min (n, j+1-i) \\ R X_{0}^{i}=\sum_{p=1}^{j-i} A \times P_{p}^{i+p} \beta_{p}^{i+p} E_{r x}^{p} \alpha_{0(j-i-p)} \\ R X_{l}^{j-i}=\sum_{p=1}^{j-i-l+1} A \times P_{p}^{i+p} \beta_{p}^{i+p} \alpha_{l(j-i-p)} E_{r x}^{p}\end{array}\right.$
The proofs for Eqs. 22 and 23 are provided in Appendices $\mathrm{B}$ and $\mathrm{C}$ respectively.

\section{B. Problem Statement}

Now that we succeed to linearly express $E_{T X}^{j+1}, E_{T X}^{i}$ and $E_{R X}^{i}, \forall 1 \leq i \leq j$, as function of $\beta^{j+1}$, we get our system of linear equations as expressed in Eq. 24.

More concisely, our previously stated system can be written as follows

$$
T X_{j+1} \beta^{j+1}=C
$$

where $C=\left(C_{0}^{i}\right)_{1<i<j}$ such that $C_{0}^{i}=T X_{0}^{i}+R X_{0}^{i}$; $\forall 1 \leq i \leq j$ and $T X_{j+1}$ is a matrix of size $(j, j+1)$ such that $T X_{j+1}=\left(T X_{i, l}\right)_{1 \leq i \leq j} \quad 1 \leq l \leq j+1$, where

$$
T X_{i, l}=\left\{\begin{array}{l}
P_{l}^{j+1} T X_{l}^{j+1}-T X_{l}^{j-i}-P_{l}^{j+1} R X_{l}^{j-i}, \\
\text { if } 1 \leq l \leq j-i \\
P_{l}^{j+1} T X_{l}^{j+1}-A\left[P_{j-i+1}^{j+1} E_{r x}^{j+1-i}+\sum_{q=1}^{i+1} \beta_{q}^{i} E_{t x}^{q}\right] \\
\text { if } l=j-i+1 \\
P_{l}^{j+1} T X_{l}^{j+1}, \text { if } j-i+1<l \leq j+1
\end{array}\right.
$$

Recall that $\beta^{j+1}$ is a column vector such that $\beta^{j+1}=\left(\beta_{l}^{j+1}\right)_{\substack{1 \leq l \leq j+1 \\ j+1}}$.

Note that, since $\sum_{l=1}^{j+1} \beta_{l}^{j+1}=1, \forall 1 \leq j+1 \leq K$ then $T X_{j+1}$ and $\beta^{j+1}$ can be respectively reduced to a square matrix of size $j$ and a vector of size $j$. Consequently, our system can be limited to only $j$ equations with $j$ unknown variables.

It is worth pointing out that, if $j+1 \leq n$ ( $n$ is the maximum number of coronas that can be reached by a sensor node), then we have a system of $j$ equations with $j$ unknown variables. Consequently, $T X_{j+1}$ is a square matrix of size $j$. Hence, we can easily resolve $T X_{j+1} \beta^{j+1}=C$ and thus the perfect uniform energy depletion is reached. However, once $j+1$ strictly exceeds $n$, then our system is actually composed of $j$ equations $\left(E^{j+1}=E^{j}=\ldots=E^{1}\right)$ with only $n$ unknown variables since $\beta_{l}^{j+1}=0$ for $l>n$. Consequently, we have much more equations than needed and hence achieving perfect uniform energy depletion is impossible. For this reason, we slightly deviate our goal to the one of minimizing the difference in energy consumption among different coronas. Consequently, in this case, we reformulate our problem as follows

$$
\begin{aligned}
& \text { Given } K, r, d_{t x-\max } \\
& \text { Find } \beta^{j+1} \\
& \min _{\beta^{j+1}}\left\|T X_{j+1} \beta^{j+1}-C\right\| \\
& \text { subject to } \\
& \sum_{l=1}^{j+1} \beta_{l}^{j+1}=1 \\
& \beta_{l}^{j+1} \geq 0, \forall 1 \leq l \leq j+1
\end{aligned}
$$

Therefore, once the iteration number exceeds $n$, the previously stated optimization problem of Eq. 27 have to be solved instead of Eq. 25. This constrained nonlinear optimization 


$$
\begin{aligned}
& \forall 1 \leq j+1 \leq K \\
& \forall 1 \leq i<j+1 \\
& E_{T X}^{j+1}=E_{T X}^{i}+E_{R X}^{i} \\
& T X_{0}^{i}+R X_{0}^{i}=\sum_{l=1}^{j+1} P_{l}^{j+1} T X_{l}^{j+1} \beta_{l}^{j+1}-\sum_{l=1}^{j-i}\left(T X_{l}^{j-i}+P_{l}^{j+1} R X_{l}^{j-i}\right) \beta_{l}^{j+1}-A\left[P_{j-i+1}^{j+1} E_{r x}^{j+1-i}+\sum_{q=1}^{i+1} \beta_{q}^{i} E_{t x}^{q}\right] \beta_{j-i+1}^{j+1}
\end{aligned}
$$

problem can be easily solved using "fmincon" function in the Matlab optimization toolbox [33].

\section{Performance Evaluation}

In this section, we present a thorough performance evaluation of our balanced routing solution along with a comparison study with the nominal communication range based data forwarding [24] $\left(d_{t x-\max }=r\right)$. The results are derived analytically. Indeed, in order to assess the performance of our proposal, we first use the underwater acoustic simulator developed in [7] in order to compute the instantaneous gain, $\tilde{G}(t)_{(i, j)}$, between any two nodes $i$ and $j$. Note that, $\tilde{G}(t)_{(i, j)}$ will be derived by assuming that the transmitter and the receiver are initially placed at the water surface within a depth of $100 \mathrm{~m}$ and using a bandwidth of $10 \mathrm{Khz}$ for communication. Moreover, we assume that the transmitter, the receiver, and the water surface randomly drift from their nominal locations by some heights $\Delta h_{T}, \Delta h_{R}$ and $\Delta h$, respectively. Besides, the distance separating the transmitter from the receiver randomly change by some $\Delta d$. Specifically, we suppose that all these displacements $\left(\Delta h_{T}, \Delta h_{R}, \Delta h\right.$ and $\left.\Delta d\right)$ are uniformly distributed, each on the interval $\pm 5 \mathrm{~m}$. Once derived, $\tilde{G}(t)_{(i, j)}$ will be used in our own developed Matlab simulator to determine numerically the average success probability $P_{j}^{i}$ for each link in the network as well as the needed transmission power $\hat{P}_{T(i, j)}$ in order to derive the optimal load weight distribution that balances the energy expenditure among all underwater sensors in the network. Algorithm 1 provides a procedure that clearly states the different phases of our work. Note that, in our simulation scenario, any failed transmission will be subject to retransmissions until the successful reception.

According to our simulation model, the underwater sensor nodes perform continuous monitoring of the supervised circular area of radius $R$. Our circular sensor field centered at the sink is partitioned into disjoint concentric coronas of constant fixed width $r$. Recall that, we assume that underwater sensors are manually deployed according to the deployment pattern of Fig. 5. The proposed deployment strategy considers a 2-dimensional shallow underwater sensor network. A set of sparse sensors is anchored to the ocean bottom and endowed with a quite long rope along with a floating buoy to help the sensor reach the surface. Each underwater sensor periodically reports with rate $A$ the locally generated data to the sink over several hops. At each hop, the traffic emanating from the local sensor must be merged with route-through traffic. Each packet is forwarded from the source to the sink by crossing coronas located in the same wedge. The parameters setting in our analysis are given in Table II.

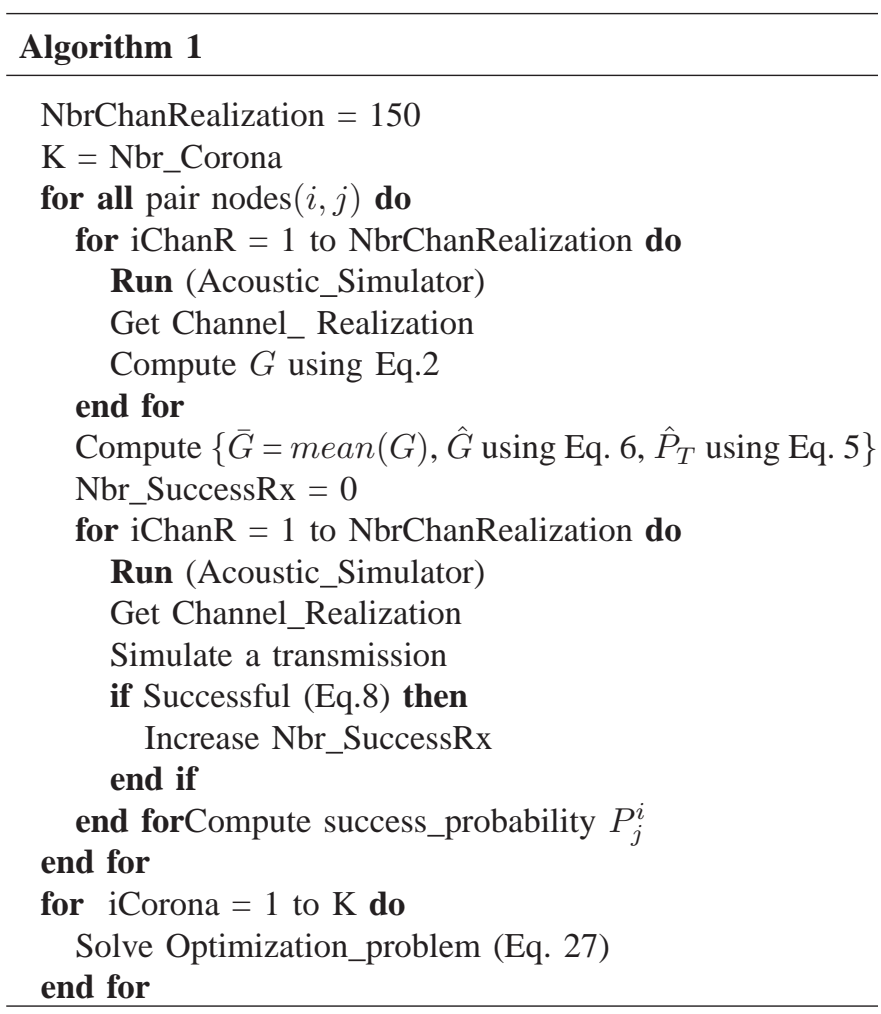

TABLE II

PARAMETERS SETTING

\begin{tabular}{|l|l|}
\hline Packet length $P_{l}$ & 1024 bits \\
\hline$S N R_{0}$ & $25 \mathrm{~dB}$ \\
\hline Initial Energy & $100 \mathrm{~J}$ \\
\hline Data Rate A & $0.08 \mathrm{packet} / \mathrm{s}$ \\
\hline Noise Power & $20 \mathrm{~dB}$ \\
\hline Bandwidth & $10 \mathrm{Khz}$ \\
\hline
\end{tabular}

We first analyze the results regarding our balanced routing strategy for a circular sensor field of radius $R=1500 \mathrm{~m}$ and corona width $r=100 \mathrm{~m}$ resulting in a total number of coronas equal to 15. Each sensor in each corona is supposed to generate 0.08 packet $/ s$. We study the impact of using variable transmission powers with different values of $n$ on both packet load and energy consumption for every corona. First, let us discover $\beta^{n}=\left(\beta_{l}^{i}\right)_{\substack{1 \leq i \leq n \\ 1 \leq i \leq 15}}$ matrix of our balanced routing scheme when $n$ is set equal to 3,5 and 7 . We want to point out that the $\beta$ matrix is derived with the purpose of evenly distributing the energy consumption among different coronas.

Table III, IV and V report the $\beta^{i}$ vectors for each corona when $n$ equals to 3,5 and 7, respectively. Accordingly, to minimize the energy consumption gap among different coronas, 
overall, the packets load should be evenly distributed among upstream coronas. Indeed, balancing the energy consumption among different coronas is a trade-off between two opposite requirements: the reception energy and the transmission energy which are the main two components of energy consumption. On one hand, since the reception energy is almost constant, hence sending over longer distance will reduce the total number of hops to reach the sink and thus the total energy consumption is minimized. On the other hand, the transmission energy is increasing with distance. More than that, the increasing rate of the transmission energy as a function of distance is also raising as shown in Fig. 4. Accordingly, it is highly preferred to send over short distances especially when the sensor is far from the sink. Reconciling these two opposites requirements leads to favoring the traffic forwarding directly to the sink, if the sink is reachable otherwise the traffic should be evenly distributed between upstream coronas as reported by Tables III, IV and V. For instance, according to Table III when $n$ is set equal to 3, much more packets should be sent to the 3-hop away corona when the sink can be directly reachable namely for coronas 1, 2 and 3. Indeed, underwater sensors in the second and third corona should send more than $90 \%$ of their accumulated traffic directly to the sink. Whereas, sensors in other coronas have to evenly distribute their traffic among all upstream coronas in order to balance the energy consumption. Similar results can be deduced for $n=5$ and $n=7$. Indeed, according to Tables IV and V, as long as the corona number is less or equal to $n$, most of the traffic should be forwarded directly to the sink using the highest possible transmission power. While farther coronas have to evenly distribute their traffic to all possible upstream coronas in order to achieve fair energy consumption among all the underwater sensors.

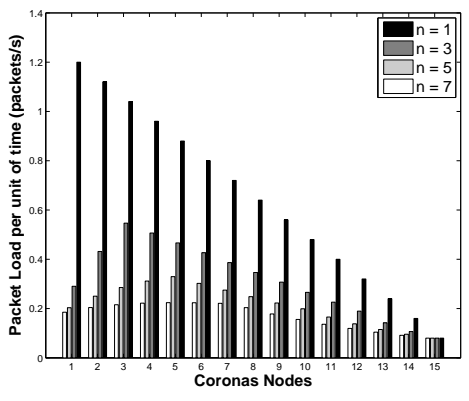

Fig. 7. Packet load distribution per corona when $R=1500 \mathrm{~m}$ and $r=$ $100 \mathrm{~m}$.

According to Tables III, IV and V the packet load distribution is shown in Fig. 7. Note that, adopting a nominal communication range based data forwarding with $d_{t x-\max }=$ $r$ (i.e. $n=1$ ) leads to a total accumulated traffic of 1.2 packet/s at sensors in corona 1 . This amount of accumulated traffic at corona 1 is highly decreased (less than 0.29 packet/s) with our balanced routing solution when $n=3$, a further decrease is achieved with $n=5$ and a further gain with $n=7$ compared to nominal based data forwarding. This increasing gain is more importantly highlighted in Fig. 8. In fact, Fig. 8 shows the energy consumption for each sensor

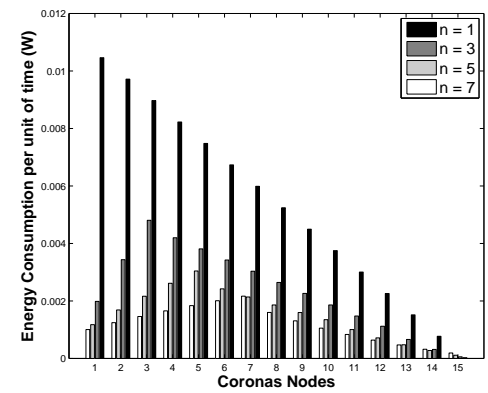

Fig. 8. Energy consumption per corona when $R=1500 \mathrm{~m}$ and $r=100 \mathrm{~m}$.

in the corresponding corona. Accordingly, a $92 \%$ of energy saving is achieved at corona 1 when $n=5$. It is worth noting that our balanced routing strategy with $n \in\{3,5,7\}$ leads to a maximum energy expenditure of $5 \mathrm{~mW}, 3 \mathrm{~mW}$, $2.2 \mathrm{~mW}$, respectively. However, according to the nominal communication range based data forwarding, a maximum energy consumption of $0.74 W$ is achieved as expected at corona 1. Consequently, an energy saving of $50 \%, 70 \%$ and $78 \%$ are accomplished thanks to our balanced routing scheme when $n \in\{3,5,7\}$, respectively. We would like to point out that the energy consumption per corona is not proportional to the packet load distribution as shown in Figs. 7 and 8 .

Indeed, as depicted in Fig. 4 the energy expenditure did not depend linearly on the transmission distance which justifies the non-proportionality between packet load distribution and the energy consumption. Consequently, balancing the energy consumption does not really mean balancing the packet load distribution.

Now, let us evaluate the gain that can be achieved by our balanced routing solution using variable transmission power over the nominal communication range based data forwarding $\left(d_{t x-\max }=r\right)$ for different field radiuses as well as different corona width. Note that this comparison study is mainly conducted in terms of energy consumption and network lifetime. From the energy depletion point of view, we consider the maximum consumed amount of energy among all coronas. Regarding network lifespan, we define the network lifetime $T$ simply as the time for the first node in the network to drain its energy budget. In other words, the network lifetime is given by

$$
T=\frac{E_{\text {init }}}{\max E(U)}
$$

where $E_{\text {init }}$ is the initial amount of energy provided to each sensor node and $E(U)$ is the consumed energy by $U$ an arbitrary underwater sensor in our field.

Figs. 9 and 10 show the energy expenditure as a function of field radius when the corona width remains fixed and equal to $r=100 \mathrm{~m}$. Recall that the energy value considered for each field radius is the maximum consumed energy among all coronas. As expected, as the field radius increases the energy consumption increases since the number of coronas grows. Consequently and as depicted in Figs. 11 and 12 the network lifetime decreases with the increase of the field radius. More importantly, our balanced routing strategy using 
TABLE III

$\beta$ MATRIX WHEN $n=3$.

\begin{tabular}{|c|l|l|l|}
\hline Corona $i$ & Corona $(i-1)$ & Corona $(i-2)$ & Corona $(i-3)$ \\
\hline Corona 1 & 1 & 0 & 0 \\
\hline Corona 2 & 0.033 & 0.97 & 0 \\
\hline Corona 3 & 0.05 & 0.05 & 0.9 \\
\hline $\begin{array}{c}\text { Corona 4 } \\
\vdots \\
\text { Corona 15 }\end{array}$ & 0.33 & 0.33 & 0.34 \\
\hline
\end{tabular}

TABLE IV

$\beta$ MATRIX WHEN $n=5$.

\begin{tabular}{|l|l|l|l|l|l|}
\hline Corona $i$ & Corona $(i-1)$ & Corona $(i-2)$ & Corona $(i-3)$ & Corona $(i-4)$ & Corona $(i-5)$ \\
\hline Corona 1 & 1 & 0 & 0 & 0 & 0 \\
\hline Corona 2 & 0.033 & 0.97 & 0 & 0 & 0 \\
\hline Corona 3 & 0.05 & 0.05 & 0.9 & 0 & 0 \\
\hline Corona 4 & 0.06 & 0.06 & 0.06 & 0.76 & 0 \\
\hline Corona 5 & 0.066 & 0.066 & 0.066 & 0.066 & 0.73 \\
\hline $\begin{array}{c}\text { Corona 6 } \\
\vdots \\
\text { Corona } 15\end{array}$ & 0.2 & 0.2 & 0.2 & 0.2 & 0.2 \\
\hline
\end{tabular}

TABLE V

$\beta$ MATRIX WHEN $n=7$.

\begin{tabular}{|c|c|c|c|c|c|c|c|}
\hline Corona $i$ & Corona $(i-1)$ & Corona $(i-2)$ & Corona $(i-3)$ & Corona $(i-4)$ & Corona $(i-5)$ & Corona $(i-6)$ & Corona $(i-7)$ \\
\hline Corona 1 & 1 & 0 & 0 & 0 & 0 & 0 & 0 \\
\hline Corona 2 & 0.033 & 0.97 & 0 & 0 & 0 & 0 & 0 \\
\hline Corona 3 & 0.05 & 0.05 & 0.9 & 0 & 0 & 0 & 0 \\
\hline Corona 4 & 0.06 & 0.06 & 0.06 & 0.82 & 0 & 0 & 0 \\
\hline Corona 5 & 0.066 & 0.066 & 0.066 & 0.066 & 0.736 & 0 & 0 \\
\hline Corona 6 & 0.07 & 0.07 & 0.07 & 0.07 & 0.07 & 0.65 & 0 \\
\hline Corona 7 & 0.07 & 0.07 & 0.07 & 0.07 & 0.07 & 0.07 & 0.58 \\
\hline $\begin{array}{c}\text { Corona } 8 \\
\vdots \\
\text { Corona } 15\end{array}$ & 0.14 & 0.14 & 0.14 & 0.14 & 0.14 & 0.14 & 0.16 \\
\hline
\end{tabular}

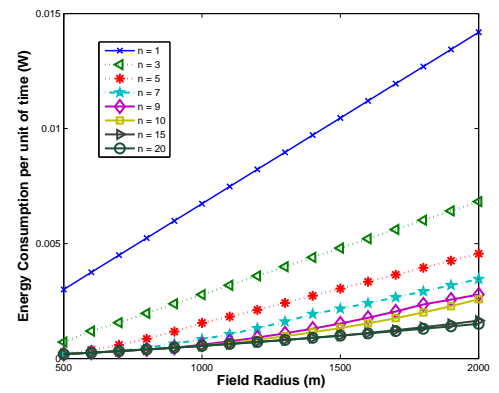

Fig. 9. Energy consumption for different field radius when $r=100 \mathrm{~m}$.

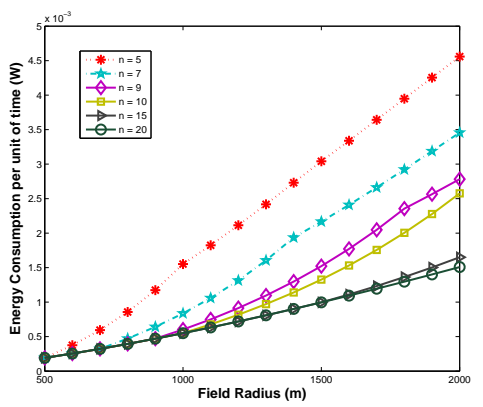

Fig. 10. Energy consumption for different field radius when $r=100 \mathrm{~m}$.

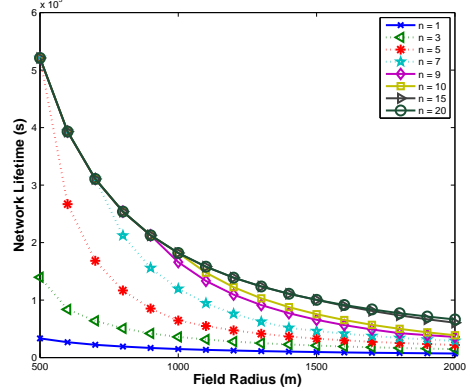

Fig. 11. Network lifetime for different field radius when $r=100 \mathrm{~m}$.

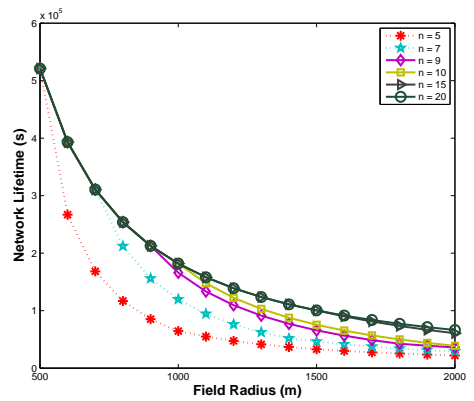

Fig. 12. Network lifetime for different field radius when $r=100 \mathrm{~m}$. 


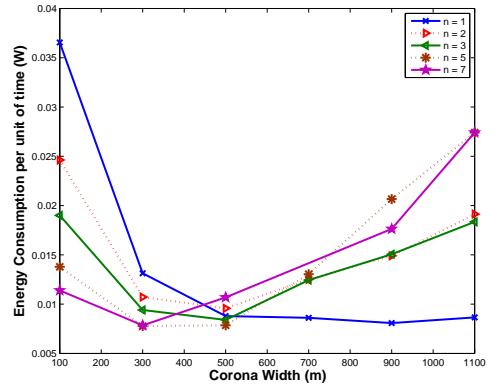

Fig. 13. Energy Consumption for various corona width when $R=5000 \mathrm{~m}$.

variable transmission powers considerably reduces the energy expenditure compared to the nominal communication range data forwarding $(n=1)$. Indeed, allowing the sensor nodes to adjust their transmission powers among $n$ different levels, leads to packet load distribution among all the upstream coronas and hence we succeed to balance the energy consumption. Note that, for a field radius of $2000 \mathrm{~m}$ our balanced routing solution achieves up to $51 \%$ of energy saving when $n=3$. A further energy saving of $34 \%$ is achieved with $n=5$ and a further saving of $66 \%$ with $n=20$. Consequently, we recommend the use of $n=5$ as a best compromise between increasing the energy savings and decreasing the number of variable transmission powers. Note that a smaller number of power levels is of interest for practical implementation.

A straightforward study showing the optimal value of $n$, in terms of energy consumption, as function of field radius when the corona width remains fixed and equal to $100 \mathrm{~m}$ was also conducted. As expected, the optimal number, $n_{\text {opt }}$, is equal to $\left\lfloor\frac{R}{r}\right\rfloor$. Indeed, by allowing each sensor to distribute its accumulated traffic among all upstream coronas and especially to directly reach the sink will highly contribute in balancing the energy consumption and thus the network lifespan is improved. That being said, can we conclude that for any values of field radius and corona width, as we extend the number of allowed transmission power levels (i.e. $n$ ) the energy savings is increased. To answer this question, we evaluate the energy consumption for various corona widths.

In order to gain more insight regarding the system performance, let us closely inspect the energy consumption as well as the network lifetime for various corona widths. To achieve this, we consider a fixed field radius of $5000 \mathrm{~m}$ while varying the corona width from $100 \mathrm{~m}$ to $1100 \mathrm{~m}$. According to Figs. 13 and 14, for each value of $n$ there is an optimal value of the corona width $C w_{\text {opt }}$ which provides the minimum energy consumption and hence the maximum network lifetime. Indeed, when the corona width increases up to $C w_{\text {opt }}$ the energy expenditure decreases. In fact, raising the corona width reduces the number of coronas and consequently the packet load is reduced. However when the corona width exceeds $C w_{\text {opt }}$ the energy expenditure increases even though the packet load is reduced. The reason behind this is the non linearity of transmission power as function of distance. Indeed, according to Fig. 4, for high value of transmission range, the transmission power is exponentially increasing leading

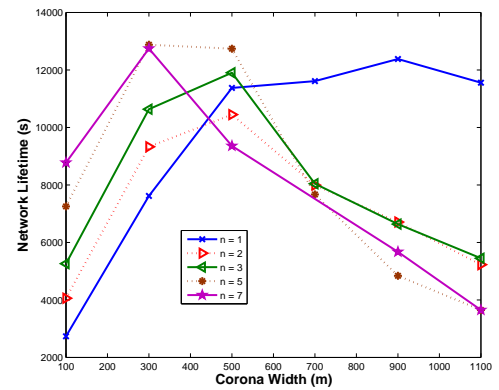

Fig. 14. Network lifetime for various corona width when $R=5000 \mathrm{~m}$.

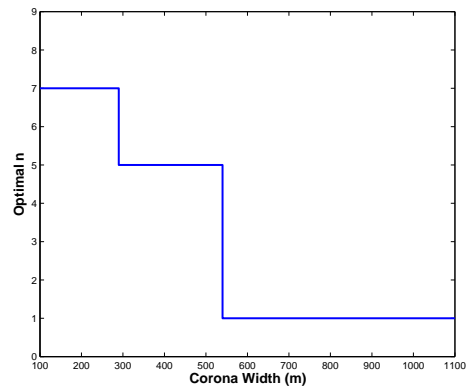

Fig. 15. Optimal $n$ value for various corona width.

consequently to high energy depletion.

Comparing our balanced routing schemes $(n>1)$ with the nominal range forwarding $(n=1)$ for different values of corona width, we observe that the schemes behave differently. Indeed, for a corona width equal to $200 \mathrm{~m}$, the minimum energy consumption is achieved by our balanced routing strategy when $n=7$ while the maximum energy depletion is achieved by the nominal communication range based data forwarding. However, for a corona width equal to $900 \mathrm{~m}$, the least energy consumption is achieved with the nominal communication range based data forwarding while the highest energy consumption is fulfilled with our balanced routing strategy when $n=5$. Consequently, there clearly exists a compromise between the number of transmission power levels $(n)$ and the energy consumption. Indeed, on one hand, as we increase $n$, we allow the traffic load to be much more distributed among all the upstream coronas and hence a balanced energy consumption is achieved. On the other hand, increasing $n$ raises the energy consumption since the farthest coronas can be reached. As a conclusion, we can state that for each corona width there is an optimal $n$ value for which the energy consumption is minimized as depicted in Fig. 15. Moreover, for each $n$, there is an optimal corona width for which the network lifetime is optimized. Combining both results, and according to Figs. 13 and 14, the maximum network lifetime is achieved by our balanced routing solution when $n=5$ for a corona width equal to $300 \mathrm{~m}$.

\section{CONCLUSION}

In underwater environments, where solar energy cannot be exploited, operating on energy constrained underwater 
sensors imposes the design of energy-efficient protocols. These protocols should be carefully designed in order to deal with the dramatically different propagation characteristics of underwater acoustic signals, such as high attenuation and bandwidthlimited channel. For these reasons, UW-ASNs require protocols that make judicious use of the limited battery budget while taking into account the unique features of the underwater channel. To this end, we proposed in this paper a routing strategy that leads to an even energy depletion among all sensors in the network and consequently an improved network lifespan. Accordingly, by allowing each underwater node to dynamically adjust its transmission power up to a predefined number of levels, we determined for each source sensor the set of possible next hops with the associated transmission power and associated load weight that lead to fair energy consumption and hence the energy sink-hole problem is overcome. To do so, we developed a comprehensive analytical model that iteratively derives for each source sensor the appropriate load weight along with the associated transmission power. Analytical results show that significant lifespan improvement is achieved by our balanced routing scheme compared to the nominal communication range based data forwarding.

\section{REFERENCES}

[1] N. Li , J. F. Martnez, J. M. Meneses Chaus and M. Eckert "A Survey on Underwater Acoustic Sensor Network Routing Protocols ", Sensors, no. 3, 2016.

[2] Salma S. Shahapur, R. Khanai "Underwater Sensor Network at physical, data link and network layer - a survey ", ICCSP 2015.

[3] M.Stojanovic and P.P.Beaujean, "Acoustic Communication ", in Springer Handbook of Ocean Engineering, M.R.Dhanak and N.I.Xiros Eds., Part B: Autonomous Ocean Vehicles and Control, T.Curtin Ed., Springer, 2016.

[4] C.-M. Chao, Y.-Z. Wang, and M.-W. Lu, "Multiple-Rendezvous Multichannel MAC Protocol Design for Underwater Sensor Networks,"IEEE Transactions on Systems, Man and Cybernetics, Part A: Systems, vol. 43, no. 1, pp.128-138, Jan. 2013.

[5] Z. Zhou, Z. Peng, J.-H. Cui, and Z. Jiang, "Handling Triple Hidden Terminal Problems for Multichannel MAC in Long-Delay Underwater Sensor Networks," IEEE Transactions on Mobile Computing, vol.11, vo.1, pp.139-154, Jan. 2012.

[6] P. Qarabaqi and M.Stojanovic, "Statistical Characterization and Computationally Efficient Modeling of a Class of Underwater Acoustic Channels ", IEEE Journal of Oceanic Engineering, Special Issue on Underwater Communications, vol. 38, no. 4, pp. 701-717, Oct. 2013

[7] The simulator package is available for download at http://millitsa.coe.neu.edu/?q=projects.

[8] S.Tadayon and M.Stojanovic, "Iterative Sparse Channel Estimation for Acoustic OFDM Systems ", in Proc. Underwater Communications and Networking (UComms'16), Lerici, Italy, August 2016.

[9] P. Qarabaqi and M. Stojanovic, "Adaptive power control for underwater acoustic channels," in Proc. IEEE Oceans'11 Europe Conference, Santander, Spain, Jun 2011

[10] A.Zoksimovski, D.Sexton, M.Stojanovic and C.Rappaport, "Underwater Electromagnetic Communications Using Conduction-Channel Characterization " , invited paper, Elsevier Journal of Physical Communication, Special issue on Advances in Underwater Communications and Networks, February 2015

[11] Y.Aval and M.Stojanovic, "Differentially Coherent Multichannel Detection of Acoustic OFDM Signals " IEEE Journal of Oceanic Engineering, vol.40, No.2, April 2015, pp.251-268.

[12] E.Zorita and M.Stojanovic, "Space-Frequency Block Coding for Underwater Acoustic Communications ", IEEE Journal of Oceanic Engineering, vol.40, No.2, April 2015, pp.303-314.

[13] A.Radosevic, R.Ahmed, T.Duman, J.Proakis and M.Stojanovic, "Adaptive OFDM modulation for Underwater Acoustic Communications: Design Considerations and Experimental Results", IEEE Journal of Oceanic Engineering, vol. 39, no. 2, Apr. 2014.
[14] W. Heinzelman, A. Chandrakashan and H. Balakrishnan, "An application-specific protocol architecture for wireless microsensor networks", IEEE Transactions on Wireless Communications, 1(4), 2002, 660-670.

[15] J. Li and P. Mohapatra, "Analytical Modeling and Mitigation Techniques for the Energy Hole Problem in Sensor Networks", Pervasive and Mobile Computing, pp. 233-254, 2007.

[16] V. Mhatre and C. Rosenberg, "Design guidelines for wireless sensor networks: communication, clustering and aggregation", Ad Hoc Networks, vol. 2, pp. 45-63, 2004.

[17] A. Wadaa, S. Olariu, L. Wilson, K. Jones, and M. Eltoweissy, "Training a sensor networks", MONET, January 2005.

[18] D. Pompili and I. F. Akyildiz, "Overview of networking protocols for underwater wireless communications", IEEE Commun. Mag., vol. 47, no. 1, pp. 97-102, Jan. 2009.

[19] M. Zorzi, P. Casari, N. Baldo, and A. Harris, "Energy-efficient routing schemes for underwater acoustic networks", IEEE J. Sel. Areas Commun., vol. 26, no. 9, pp. 1754-1766, Dec. 2008.

[20] D. Pompili, T. Melodia, and I. F. Akyildiz, "Routing algorithms for delay-insensitive and delay-sensitive applications in underwater sensor networks", in Proc. 12th ACM Annu. Int. Conf. Mobile Comput. Netw., 2006, pp. 298-309.

[21] J. M. Jornet, M. Stojanovic, and M. Zorzi, "Focused beam routing protocol for underwater acoustic networks", in Proc. 3rd ACM Int. Workshop Underwater Netw., Sep. 2008, pp. 75-82.

[22] Z. Zhou and J. H. Cui, "Energy efficient multi-path communication for time-critical applications in underwater sensor networks", in Proc. 9th ACM Int. Symp. Mobile Ad Hoc Netw. Comput., May 2008, pp. 221230.

[23] D. Pompili and I. F. Akyildiz, "A cross-layer communication solution for multimedia applications in underwater acoustic sensor networks", in Proc. 5th IEEE Int. Conf. Mobile Ad Hoc Sens. Syst., Oct. 2008, pp. 275-284

[24] Habib M. Ammari and Sajal K. Das, "Promoting Heterogeneity, Mobility, and Energy-Aware Voronoi Diagram in Wireless Sensor Networks", IEEE Transactions on Parralel and Distributed Systems, vol. 19, no. 7, July 2008.

[25] F. Bouabdallah, N. Bouabdallah, and R. Boutaba, "On Balancing Energy Consumption in Wireless Sensor Networks", IEEE Transactions on Vehicular Technology, vol. 58 , no. 6, pp 2909-2924, July 2009.

[26] A. Boukerche, I. Chatzigiannakis, and S. Nikoletseas, "A New Energy Efficient and Fault-Tolerant Protocol for Data Propagation in Smart Dust Networks Using Varying Transmission Range", Computer Comm., vol. 4, no. 29, pp. 477-489, Feb. 2006.

[27] W. Guo, Z. Liu, and G. Wu, "An Energy-Balanced Transmission Scheme for Sensor Networks", Poster, Proc. First ACM Int'l Conf. Embedded Networked Sensor Systems (SenSys '03), pp. 300-301, 2003.

[28] P. Leone, S. Nikoletseas, and J. Rolim, "An Adaptive Blind Algorithm for Energy Balanced Data Propagation in Wireless Networks", Proc. First IEEE Int'l Conf. Distributed Computing in Sensor Systems (DCOSS '05), pp. 35-48, 2005

[29] S. Olariu and I. Stojmenovic, "Design Guidelines for Maximizing Lifetime and Avoiding Energy Holes in Sensor Networks with Uniform Distribution and Uniform Reporting”, Proc. IEEE INFOCOM '06, pp. $1-12,2006$

[30] J. Lian, K. Naik, and G. Agnew, "Data Capacity Improvement of Wireless Sensor Networks Using Non-Uniform Sensor Distribution", Int'1 J. Distributed Sensor Networks, vol. 2, no. 2, pp. 121-145, Apr.June 2006.

[31] O. Powell, P. Leone, and J. Rolim, "Energy Optimal Data Propagation in Wireless Sensor Networks", J. Parallel and Distributed Computing, vol. 3, no. 67, pp. 302-317, Mar. 2007.

[32] M. Stojanovic, "On the relationship between capacity and distance in an underwater acoustic communication channel", ACM SIGMOBILE Mobile Comput. Commun. Rev., vol. 11, no. 4, pp. 34-43, Oct. 2007.

[33] MATLAB help, R2009a, Available: http:// www.mathworks.com 


\section{APPENDIX A}

\section{PROOF OF THEOREM 1}

We prove Theorem 1 by mathematical induction.

Initial Step. At each iteration $j+1$, corona $B_{j+1}$ is newly added where $A_{j+1}=A$. Thus, the cumulative traffic at corona $B_{j}$ will increase to $A_{j}=A+P_{l}^{j+1} \beta_{1}^{j+1} A_{j+1}$ and hence it can be written as $A_{j}=A \times\left(1+P_{1}^{j+1} \beta_{1}^{j+1}\right)$. Consequently, $\alpha_{00}$ equals 1 and $\alpha_{10}$ too.

Inductive Step. Let us assume that

$$
\begin{aligned}
& \forall p ; 0 \leq p \leq k \\
& A_{j-p}=\left[\alpha_{0 p}+\sum_{l=1}^{p+1} \alpha_{l p} P_{l}^{j+1} \beta_{l}^{j+1}\right] \times A
\end{aligned}
$$

where $\beta_{l}^{j+1}=0$ if $l>\min (n, p+1)$.

is true. Then, we need to prove that our assumption is also true for $A_{j-(k+1)}$.

By definition, $A_{j-(k+1)}$ can be expressed as follows

$$
\begin{aligned}
& A_{j-(k+1)}=A+\sum_{m=1}^{k+1} P_{m}^{j-(k+1-m)} \beta_{m}^{j-(k+1-m)} A_{j-(k+1-m)} \\
& +P_{k+2}^{j+1} \beta_{k+2}^{j+1} A_{j+1}
\end{aligned}
$$

Since we assume that our assumption is true up to corona $B_{j-k}$, then $A_{j-(k+1-m)}$ can be substituted by Eq. 29 and hence we can express $A_{j-(k+1)}$ as follows

$$
\begin{aligned}
A_{j-(k+1)}=\left\{\begin{array}{l}
A+A P_{k+2}^{j+1} \beta_{k+2}^{j+1}+A \sum_{m=1}^{k+1} P_{m}^{j-(k+1-m)} \beta_{m}^{j-(k+1-m)} \\
\times\left(\alpha_{0(k+1-m)}+\sum_{l=1}^{k+2-m} \alpha_{l(k+1-m)} P_{l}^{j+1} \beta_{l}^{j+1}\right)
\end{array}\right. \\
=A \times\left(\begin{array}{l}
1+ \\
\sum_{m=1}^{k+1} \alpha_{0(k+1-m)} P_{m}^{j-(k+1-m)} \beta_{m}^{j-(k+1-m)} \\
+\left(\sum_{m=1}^{k+1} \sum_{l=1}^{k+2-m} \alpha_{l(k+1-m)} P_{m}^{j-(k+1-m)}\right. \\
\beta_{m}^{j-(k+1-m)} P_{l}^{j+1} \beta_{l}^{j+1}+P_{k+2}^{j+1} \beta_{k+2}^{j+1}
\end{array}\right) \text { Note }
\end{aligned}
$$

Note that $\left(1+\sum_{m=1}^{k+1} \alpha_{0(k+1-m)} P_{m}^{j-(k+1-m)} \beta_{m}^{j-(k+1-m)}\right)$ $A\left(\sum_{q=1}^{i+1} \beta_{q}\right.$ respectively, are constants (not depending on $\beta^{j+1}$ ).

$$
\begin{aligned}
& E_{T X}^{i}=\left(\sum_{q=1}^{i+1} \beta_{q}^{i} E_{t x}^{q}\right) A_{j-(j-i)} \\
& =\left\{\begin{array}{l}
\left(\sum_{q=1}^{i+1} \beta_{q}^{i} E_{t x}^{q}\right) \times A \\
\times\left[\alpha_{0(j-i)}+\sum_{l=1}^{j-i+1} \alpha_{l(j-i)} P_{l}^{j+1} \beta_{l}^{j+1}\right]
\end{array}\right. \\
& =\left\{A\left(\sum_{q=1}^{i+1} \beta_{q}^{i} E_{t x}^{q}\right) \alpha_{0(j-i)}\right. \\
& +\sum_{l=1}^{j-i+1} A\left(\sum_{q=1}^{i+1} \beta_{q}^{i} E_{t x}^{q}\right) \alpha_{l(j-i)} P_{l}^{j+1} \beta_{l}^{j+1}
\end{aligned}
$$

Hence we get

is simply $\alpha_{0(k+1)}$ and $\left(\sum_{m=1}^{k+2-l} \alpha_{l(k+1-m)} P_{m}^{j-(k+1-m)} \beta_{m}^{j-(k+1-m)}\right)$

$$
E_{T X}^{i}=T X_{0}^{i}+\sum_{l=1}^{j-i+1} P_{l}^{j+1} T X_{l}^{j-i} \beta_{l}^{j+1}
$$
is simply $\alpha_{l(k+1)}$.

Consequently, $A_{j-(k+1)}$ can be expressed as follows

$$
\begin{aligned}
A_{j-(k+1)}= & A\left(\alpha_{0 k+1}+\sum_{l=1}^{k+1} \alpha_{l(k+1)} P_{l}^{j+1} \beta_{l}^{j+1}+P_{k+2}^{j+1} \beta_{k+2}^{j+1}\right) \\
& =A\left(\alpha_{0 k+1}+\sum_{l=1}^{k+2} \alpha_{l(k+1)} P_{l}^{j+1} \beta_{l}^{j+1}\right)
\end{aligned}
$$

where $\beta_{l}^{j+1}=0$ if $l>\min (n, j-i+1)$ which concludes the proof.

Therefore, our assumption is also true for $A_{j-(k+1)}$ so that the proof completes. 


\section{APPENDIX C}

PROOF OF EQ. 23

Similar to Eq. 22 proof explained previously, we can linearly express $E_{R X}^{i}$ as function of $\beta^{j+1}$. Observe that $E_{R X}^{i}$ is simply $E_{R X}^{j-(j-i)}$. Consequently, it can be written as follows

$$
\begin{aligned}
E_{R X}^{i}= & \sum_{p=1}^{j+1-i} P_{p}^{i+p} \beta_{p}^{i+p} A_{i+p} E_{r x}^{p} \\
& =\sum_{p=1}^{j-i} P_{p}^{i+p} \beta_{p}^{i+p} A_{i+p} E_{r x}^{p}+P_{j-i+1}^{j+1} \beta_{j-i+1}^{j+1} A E_{r x}^{j+1-i}
\end{aligned}
$$

where $\beta_{p}^{i+p}=0$ if $p>\min (n, j+1-i)$.

By substituting $A_{i+p}$ with the appropriate expression of theorem 1, we succeed to get a linear expression of $E_{R X}^{i}$ as function of $\beta^{j+1}$ as follows

$$
E_{R X}^{i}=\left\{\begin{array}{l}
\sum_{p=1}^{j-i} A P_{p}^{i+p} \beta_{p}^{i+p} E_{r x}^{p} \\
{\left[\alpha_{0(j-i-p)}+\sum_{l=1}^{j-i-p+1} P_{l}^{j+1} \alpha_{l(j-i-p)} \beta_{l}^{j+1}\right]} \\
+P_{j-i+1}^{j+1} \beta_{j-i+1}^{j+1} A E_{r x}^{j+1-i} \\
\sum_{p=1}^{j-i} P_{p}^{i+p} A \beta_{p}^{i+p} E_{r x}^{p} \alpha_{0(j-i-p)} \\
+\sum_{p=1}^{j-i j-i-p+1} \sum_{l=1}^{j+1} A \beta_{p}^{i+p} \alpha_{l(j-i-p)} E_{r x}^{p} P_{l}^{j+1} \beta_{l}^{j+1} \\
+P_{j-i+1}^{j+1} \beta_{j-i+1}^{j+1} A E_{r x}^{j+1-i}
\end{array}\right.
$$

Observe that, by performing indices permutation, $\left(\sum_{p=1}^{j-i} \sum_{l=1}^{j-i-p+1} A P_{p}^{i+p} \beta_{p}^{i+p} \alpha_{l(j-i-p)} E_{r x}^{p} P_{l}^{j+1} \beta_{l}^{j+1}\right)$ is simply equal to $\left(\sum_{l=1}^{j-i} \sum_{p=1}^{j-i-l+1} A P_{p}^{i+p} \beta_{p}^{i+p} \alpha_{l(j-i-p)} E_{r x}^{p} P_{l}^{j+1} \beta_{l}^{j+1}\right)$. $\begin{array}{ccc}\text { Moreover, } & \text { it is worth pointing out } \\ \text { that } & \left(\sum_{p=1}^{j-i} P_{p}^{i+p} A \beta_{p}^{i+p} E_{r x}^{p} \alpha_{0(j-i-p)}\right) & \text { and }\end{array}$ $\left(\sum_{p=1}^{j-i-l+1} P_{p}^{i+p} A \beta_{p}^{i+p} \alpha_{l(j-i-p)} P_{l}^{j+1} E_{r x}^{p}\right)$ are independents of $\beta^{j+1}$ thus we call them $R X_{0}^{i}$ and $R X_{l}^{j-i}$ respectively.

Consequently, we get

$$
\begin{aligned}
& E_{R X}^{i}=R X_{0}^{i}+\sum_{l=1}^{j-i} P_{l}^{j+1} R X_{l}^{j-i} \beta_{l}^{j+1}+ \\
& P_{j-i+1}^{j+1} \beta_{j-i+1}^{j+1} A E_{r x}^{j+1-i} ;
\end{aligned}
$$

where $\beta_{p}^{i+p}=0$; if $p>\min (n, j+1-i)$ which completes the proof.
Fatma Bouabdallah received a B.S. degree in computer engineering from Ecole Nationale des Sciences de l'Informatique (ENSI), Tunis, Tunisia, in 2005, and the M.S. degree in network and computer science from the University of Paris VI, France, in 2006. She attended the doctoral program at the Institut National de Recherche en Informatique et en Automatique, Rennes, France from October 2006 to November 2008. In November 2008, she received her Ph.D. degree in computer Science. In 2010, she was with the University of Waterloo, Canada, as a Postdoctoral Fellow. She is currently an assistant Professor in King AbdulAziz University, Jeddah, Saudi Arabia. Her research interests include wireless sensor networks, under-water acoustic sensor networks, crosslayer design, modeling and performance evaluation of computer networks.

Chaima Zidi received his Computer Science Engineering and Master degrees from the National College of Computer Science (ENSI, Tunisia) in respectively 2010 and 2011. And at the beginning of 2013, she joined Faculty of Computing and Information Technology (Jeddah, Saudi Arabia). Now, she prepare a Ph.D in Underwater Acoustic Sensor Networks within LIPADE Laboratory (Paris, France).

Raouf Boutaba received the M.Sc. and Ph.D. degrees in computer science from the University Pierre et Marie Curie, Paris, in 1990 and 1994, respectively. He is currently a professor of computer science at the University of Waterloo. His research interests include resource and service management in networks and distributed systems. He is the founding editor in chief of the IEEE Transactions on Network and Service Management (2007-2010) and on the editorial boards of other journals. He has received several best paper awards and other recognitions such as the Premiers Research Excellence Award, the IEEE Hal Sobol, Fred W. Ellersick, Joe LociCero, Dan Stokesbury, Salah Aidarous Awards, and the McNaughton Gold Medal. He is a fellow of the IEEE, the Engineering Institute of Canada, and the Canadian Academy of Engineering. 